\title{
On a class of nonlinear elliptic equations in Hilbert spaces
}

\author{
by Piotr FijaŁkowski (Łódź)
}

\begin{abstract}
We consider elliptic nonlinear equations in a separable Hilbert space and
\end{abstract} their solutions in spaces of Sobolev type.

1. Introduction. We study equations of the form

$$
P(D) u=F\left(x,\left(\partial^{\alpha} u\right)\right) \quad(D=-i \partial),
$$

with a strongly elliptic polynomial $P$ of $n$ variables, defined for $u: \mathbb{R}^{n} \rightarrow H$, where $H$ is a separable Hilbert space. The equations are understood in a weak sense (see Definition 2). We make assumptions giving an a priori bound for solutions in a space of Sobolev type. As an example, we consider assumptions of Bernstein type. Assumptions of this kind appear in the papers [1], [5] concerning equations on a bounded interval, and in [8], [3] and [4] concerning equations on the half-line, on the line and in $\mathbb{R}^{n}$, respectively.

\section{Spaces of Sobolev type}

Definition 1. We denote by $\mathcal{H}^{s}=\mathcal{H}^{s}\left(\mathbb{R}^{n}\right)$, for $s \in \mathbb{R}$, the Sobolev space of real tempered distributions $u$ such that

$$
\|u\|_{s}^{2}:=(2 \pi)^{-n} \int|\mathcal{F} u(\xi)|^{2}\left(1+|\xi|^{2}\right)^{s} d \xi<\infty
$$

where $\mathcal{F}$ stands for the Fourier transform.

Remark 1. Definition 1 may be used for both real and complex Sobolev spaces, depending on whether we consider real or complex functions and distributions.

$\mathcal{H}^{s}$ is a Hilbert space with the scalar product

$$
\langle u, w\rangle_{s}:=(2 \pi)^{-n} \int(\mathcal{F} u)(\xi) \overline{(\mathcal{F} w)(\xi)}\left(1+|\xi|^{2}\right)^{s} d \xi
$$


in the complex case or

$$
\langle u, w\rangle_{s}:=\operatorname{Re}(2 \pi)^{-n} \int(\mathcal{F} u)(\xi) \overline{(\mathcal{F} w)(\xi)}\left(1+|\xi|^{2}\right)^{s} d \xi
$$

in the real case.

We denote the local Sobolev space by $\mathcal{H}_{\text {loc }}^{s}=\mathcal{H}_{\text {loc }}^{s}\left(\mathbb{R}^{n}\right)$ and treat it as a Fréchet space in the standard way (see for example [6]).

Note two important lemmas.

LEMma 1. The embedding $\mathcal{H}_{\mathrm{loc}}^{s} \rightarrow \mathcal{H}_{\mathrm{loc}}^{s^{\prime}}$, for $s>s^{\prime}$, is completely continuous.

The proof is in [6], Theorem 10.1.27.

LEMMA 2. If $u \in \mathcal{H}^{s}$ then any $\partial^{\alpha} u$, for $|\alpha|<s-n / 2$, is a continuous bounded function and there exists a constant $C$ such that

$$
\sup _{x \in \mathbb{R}^{n}} \sup _{|\alpha|<s-n / 2}\left|\partial^{\alpha} u(x)\right| \leq C\|u\|_{s} .
$$

Pr o of. See [6], Corollary 7.9.4. One can obtain inequality (2) by a standard calculation.

Assume that $\left(H,\langle\cdot, \cdot\rangle_{H}\right)$ is a complex Hilbert space. Let $L^{2}\left(\mathbb{R}^{n}, H\right)$ be the Hilbert space of measurable functions $u: \mathbb{R}^{n} \rightarrow H$ for which

$$
\|u\|_{L^{2}\left(\mathbb{R}^{n}, H\right)}^{2}:=\int\|u(x)\|_{H}^{2} d x<\infty .
$$

The scalar product in $L^{2}\left(\mathbb{R}^{n}, H\right)$ is defined by

$$
\langle u, w\rangle_{L^{2}\left(\mathbb{R}^{n}, H\right)}:=\int\langle u(x), w(x)\rangle_{H} d x .
$$

Let $\left(e_{\gamma}\right)_{\gamma \in \Gamma}$ be a complete orthonormal system in $H$. For $u \in L^{2}\left(\mathbb{R}^{n}, H\right)$, let

$$
u_{\gamma}(x):=\left\langle u(x), e_{\gamma}\right\rangle_{H} .
$$

By the Bessel inequality, for any finite set $\Gamma^{\prime} \subset \Gamma$, we have

$$
\begin{aligned}
\|u\|_{L^{2}\left(\mathbb{R}^{n}, H\right)}^{2} & =\int\|u(x)\|_{H}^{2} d x \geq \int \sum_{\gamma \in \Gamma^{\prime}}\left|u_{\gamma}(x)\right|^{2} d x \\
& =\sum_{\gamma \in \Gamma^{\prime}} \int\left|u_{\gamma}(x)\right|^{2} d x=\sum_{\gamma \in \Gamma^{\prime}}\left\|u_{\gamma}\right\|_{0}^{2} .
\end{aligned}
$$

Hence at most countable many $u_{\gamma}$ are nonzero outside a set of measure zero. From the Lebesgue theorem and the Parseval equality, we have

$$
\|u\|_{L^{2}\left(\mathbb{R}^{n}, H\right)}^{2}=\sum_{\gamma \in \Gamma}\left\|u_{\gamma}\right\|_{0}^{2} .
$$


We define the Fourier transform for $L^{2}\left(\mathbb{R}^{n}, H\right)$ by means of the Fourier transform in $L^{2}\left(\mathbb{R}^{n}, \mathbb{C}\right)$ :

$$
\mathcal{F} u:=\sum_{\gamma \in \Gamma}\left(\mathcal{F} u_{\gamma}\right) e_{\gamma} .
$$

One can verify that this definition is independent of the choice of a complete orthonormal system $\left(e_{\gamma}\right)_{\gamma \in \Gamma}$ and that $\mathcal{F}$ is an isomorphism of $L^{2}\left(\mathbb{R}^{n}, H\right)$ onto itself and, by (3),

$$
\|u\|_{L^{2}\left(\mathbb{R}^{n}, H\right)}^{2}=(2 \pi)^{-n}\|\mathcal{F} u\|_{L^{2}\left(\mathbb{R}^{n}, H\right)}^{2}
$$

for any $u \in L^{2}\left(\mathbb{R}^{n}, H\right)$.

For $s \geq 0$, we define the space $\mathcal{H}^{s}\left(\mathbb{R}^{n}, H\right)$ to be $\left\{u \in L^{2}\left(\mathbb{R}^{n}, H\right):\|u\|_{\mathcal{H}^{s}\left(\mathbb{R}^{n}, H\right)}^{2}:=(2 \pi)^{-n} \int\|\mathcal{F} u(\xi)\|_{H}^{2}\left(1+|\xi|^{2}\right)^{s} d \xi<\infty\right\}$. $\mathcal{H}^{s}\left(\mathbb{R}^{n}, H\right)$ is a Hilbert space with the scalar product

$$
\langle u, w\rangle_{\mathcal{H}^{s}\left(\mathbb{R}^{n}, H\right)}:=(2 \pi)^{-n} \int\langle\mathcal{F} u(\xi), \mathcal{F} w(\xi)\rangle_{H}\left(1+|\xi|^{2}\right)^{s} d \xi .
$$

In the case of a real Hilbert space $H$, we mean by $\mathcal{H}^{s}\left(\mathbb{R}^{n}, H\right)$ the real Hilbert space

$$
\left\{u \in \mathcal{H}^{s}\left(\mathbb{R}^{n}, H+i H\right): u(x) \in H \text { for almost every } x \in \mathbb{R}^{n}\right\} .
$$

We shall use derivatives of $\mathcal{H}^{s}\left(\mathbb{R}^{n}, H\right)$ functions in the following weak sense:

Definition 2. Let $u \in \mathcal{H}^{s}\left(\mathbb{R}^{n}, H\right), \alpha \in \mathbb{N}^{n},|\alpha| \leq s$. We denote by $\partial^{\alpha} u$ an element of $L^{2}\left(\mathbb{R}^{n}, H\right)$ such that

$$
\left\langle\partial^{\alpha} u(\cdot), h\right\rangle_{H}=\partial^{\alpha}\langle u(\cdot), h\rangle_{H} \quad \text { for any } h \in H .
$$

Note that if $u(x)=\sum_{\gamma \in \Gamma} u_{\gamma}(x) e_{\gamma}$, then $\partial^{\alpha} u(x)=\sum_{\gamma \in \Gamma} \partial^{\alpha} u_{\gamma}(x) e_{\gamma}$.

3. Existence theorem for a single equation. We shall construct a solution of an equation of the form (1) in the space $\mathcal{H}^{t}\left(\mathbb{R}^{n}, H\right)$ by approximation by a sequence of solutions of adapted problems "with values in finite-dimensional spaces". The following lemma plays the basic role in this construction:

Lemma 3. Every sequence $\left(u_{k}\right)$ in $\mathcal{H}^{s}\left(\mathbb{R}^{n}, H\right)$ weakly convergent to $u \in$ $\mathcal{H}^{s}\left(\mathbb{R}^{n}, H\right)$ contains a subsequence $\left(u_{k_{l}}\right)$ for which the sequences $\left(\partial^{\alpha} u_{k_{l}}(x)\right)$ weakly converge in $H$ to $\left(\partial^{\alpha} u\right)(x)$ for a.e. $x$ and $|\alpha|<s-n / 2$.

P r o of. The weak convergence of $\left(u_{k}\right)$ implies its boundedness:

$$
\left\|u_{k}\right\|_{\mathcal{H}^{s}\left(\mathbb{R}^{n}, H\right)} \leq M
$$

for some constant $M$. 
The essential ranges of the functions $u_{k}, k=1,2, \ldots$ (without the values on a set of measure zero) are contained in a separable subspace of $H$ (see Section 2). Hence we can assume that $H$ is separable. Let $\left(e_{\gamma}\right)_{\gamma \in \Gamma}$ be a complete orthonormal system in $H$ (at most countable).

The weak convergence of $\left(u_{k}\right)$ in $\mathcal{H}^{s}\left(\mathbb{R}^{n}, H\right)$ implies that

$$
\left\langle\partial^{\alpha} u_{k}(\cdot), e_{\gamma}\right\rangle_{H} \rightarrow\left\langle\partial^{\alpha} u(\cdot), e_{\gamma}\right\rangle_{H} \quad \text { weakly in } L^{2}
$$

for any $|\alpha| \leq s$ and $\gamma \in \Gamma$.

From (7), we have

$$
\left\|\left\langle u_{k}(\cdot), e_{\gamma}\right\rangle_{H}\right\|_{s} \leq M
$$

Making use of (9) and Lemma 1, we construct by the diagonal method a subsequence $\left(u_{k_{l}}\right)$ such that, for any $\gamma \in \Gamma$,

$$
\left\langle u_{k_{l}}(\cdot), e_{\gamma}\right\rangle_{H} \rightarrow w_{\gamma} \in \mathcal{H}_{\mathrm{loc}}^{s-n / 2} \quad \text { in } \mathcal{H}_{\mathrm{loc}}^{s-n / 2} .
$$

For $|\alpha| \leq s-n / 2$, we have

$$
\left\langle\partial^{\alpha} u_{k_{l}}(\cdot), e_{\gamma}\right\rangle_{H} \rightarrow \partial^{\alpha} w_{\gamma} \quad \text { in } L_{\text {loc }}^{2} \text {, for any } \gamma \in \Gamma \text {. }
$$

Comparing (8) and (10) for $\alpha=(0, \ldots, 0)$, we obtain $\left\langle u(x), e_{\gamma}\right\rangle_{H}=$ $w_{\gamma}(x)$ for a.e. $x$, hence $\left\langle u_{k_{l}}(\cdot), e_{\gamma}\right\rangle_{H} \rightarrow\left\langle u(\cdot), e_{\gamma}\right\rangle_{H}$ in $\mathcal{H}_{\mathrm{loc}}^{s-n / 2}$. By the diagonal method, we construct a subsequence (denoted again by $\left(u_{k_{l}}\right)$ for simplicity of notation) such that

$$
\left\langle\partial^{\alpha} u_{k_{l}}(x), e_{\gamma}\right\rangle_{H} \rightarrow\left\langle\partial^{\alpha} u(x), e_{\gamma}\right\rangle_{H} \quad \text { for a.e. } x,
$$

for any $|\alpha| \leq s-n / 2$ and $\gamma \in \Gamma$. We shall show that this is the desired subsequence. By (11), it is sufficient to show that, for $|\alpha|<s-n / 2$, the set $\left\{\left\|\partial^{\alpha} u_{k_{l}}(x)\right\|\right\}$ is bounded for a.e. $x$. From (2) and (7), we have

which ends the proof.

$$
\begin{aligned}
\left\|\partial^{\alpha} u_{k_{l}}(x)\right\|^{2} & =\sum_{\gamma \in \Gamma}\left|\left\langle\partial^{\alpha} u_{k_{l}}(x), e_{\gamma}\right\rangle_{H}\right|^{2} \\
& \leq C^{2} \sum_{\gamma \in \Gamma}\left\|\left\langle u_{k_{l}}(\cdot), e_{\gamma}\right\rangle_{H}\right\|_{s}^{2} \\
& =C^{2}\left\|u_{k_{l}}\right\|_{\mathcal{H}^{s}\left(\mathbb{R}^{n}, H\right)}^{2} \leq C^{2} M^{2},
\end{aligned}
$$

We now formulate and prove the main

THEOREM 1. Let $H$ be a real, infinite-dimensional separable Hilbert space, and $\left(e_{\gamma}\right)_{\gamma=1,2, \ldots}$ a complete orthonormal system in $H$. Let $H_{p}$ denote the space generated by $\left\{e_{\gamma}: \gamma=1, \ldots, p\right\}$ and let $R_{p}: H \rightarrow H_{p}$ be the orthonormal projector onto $H_{p}$. Let $P$ be a polynomial of $n$ variables and degree $T$ such that the polynomial $P(-i \partial)$ of the variable $\partial$ has real coefficients and satisfies the condition

$$
1+|\xi|^{T} \leq C P(\xi), \quad \xi \in \mathbb{R}^{n} .
$$


Fix $t \in[0, T[$ and set

$$
m:=\sum_{0 \leq l<t-n / 2} n^{l} .
$$

Let $F: \mathbb{R}^{n} \times \mathbb{R}^{m} \rightarrow H$ satisfy the Carathéodory condition of the following form: $F(x, \cdot)$ is sequentially continuous in the weak topologies of $H^{m}$ and $H$ for a.e. $x$, and $F\left(\cdot,\left(v_{\alpha}\right)_{|\alpha|<t-n / 2}\right)$ is measurable for all $\left(v_{\alpha}\right)_{|\alpha|<t-n / 2} \in H^{m}$.

Suppose that for any bounded set $K \subset \mathbb{R}^{n} \times H^{m}$ there exists a function $h_{K} \in L^{2}\left(\mathbb{R}^{n}\right)$ such that

$$
\left\|F\left(x,\left(v_{\alpha}\right)_{|\alpha|<t-n / 2}\right)\right\| \leq h_{K}(x) \quad \text { for a.e. } x
$$

for $\left(x,\left(v_{\alpha}\right)_{|\alpha|<t-n / 2}\right) \in K\left(\|\cdot\|\right.$ denotes the norm in $H^{k}$ for any $\left.k \in \mathbb{N}\right)$. Assume that there is a sequence of open bounded sets $U_{1} \subset U_{2} \subset \ldots$ with $\bigcup U_{j}=\mathbb{R}^{n}$ and a constant $M$ such that no equation

$$
P(D) u=\lambda R_{p} F_{j}\left(x,\left(\partial^{\alpha} u\right)_{|\alpha|<t-n / 2}\right), \quad j=1,2, \ldots, \lambda \in[0,1],
$$

with

$$
F_{j}\left(x,\left(v_{\alpha}\right)_{|\alpha|<t-n / 2}\right):= \begin{cases}F\left(x,\left(v_{\alpha}\right)_{|\alpha|<t-n / 2}\right) & \text { for } x \in U_{j}, \\ 0 & \text { for } x \notin U_{j}\end{cases}
$$

has a solution in the set

$$
\left\{u \in \mathcal{H}^{t}\left(\mathbb{R}^{n}, H_{p}\right):\|u\|_{\mathcal{H}^{t}\left(\mathbb{R}^{n}, H\right)}>M\right\}, \quad p=1,2, \ldots
$$

Under these assumptions the equation

$$
P(D) u=F\left(x,\left(\partial^{\alpha} u\right)_{|\alpha|<t-n / 2}\right),
$$

understood in the sense of Definition 2 , has a solution $u \in \mathcal{H}^{t}\left(\mathbb{R}^{n}, H\right)$ for which

$$
\|u\|_{\mathcal{H}^{t}\left(\mathbb{R}^{n}, H\right)} \leq M
$$

Proof. Consider the equations

$$
P(D) u=R_{p} F\left(x,\left(\partial^{\alpha} u\right)_{|\alpha|<t-n / 2}\right), \quad p=1,2, \ldots
$$

Treating $H_{p}$ as $\mathbb{R}^{p}$, we conclude, from the assumptions of the theorem, that equation (17) has a solution $u_{p} \in \mathcal{H}^{t}\left(\mathbb{R}^{n}, H_{p}\right)$ for any $p$ (see [4], Theorem 2). We have

$$
\left\|u_{p}\right\|_{\mathcal{H}^{t}\left(\mathbb{R}^{n}, H\right)} \leq M, \quad p=1,2, \ldots
$$

By the Eberlein-Shmul'yan theorem, the sequence $\left(u_{p}\right)$ contains a subsequence $\left(u_{p_{k}}\right)$ weakly convergent to some $u \in \mathcal{H}^{t}\left(\mathbb{R}^{n}, H\right)$ and

$$
\|u\|_{\mathcal{H}^{t}\left(\mathbb{R}^{n}, H\right)} \leq M
$$

By Lemma 3, we may assume that the sequences $\left(\partial^{\alpha} u_{p_{k}}(x)\right),|\alpha|<t-n / 2$, weakly converge in $H$ to the corresponding $\partial^{\alpha} u(x)$ for a.e. $x$. 

that

We shall prove that $u$ is a solution of equation (16). We have to show

$$
P(D)\langle u(\cdot), h\rangle_{H}=\left\langle F\left(\cdot,\left(\partial^{\alpha} u(\cdot)\right)_{|\alpha|<t-n / 2}\right), h\right\rangle_{H}
$$

in $\mathcal{D}^{\prime}$ for any $h \in H$. We know that

$$
P(D)\left\langle u_{p_{k}}(\cdot), h\right\rangle_{H}=\left\langle R_{p_{k}} F\left(\cdot,\left(\partial^{\alpha} u_{p_{k}}(\cdot)\right)_{|\alpha|<t-n / 2}\right), h\right\rangle_{H}
$$

in $\mathcal{D}^{\prime}$. We shall prove that (17) follows from (19) by passing to the limit in $\mathcal{D}^{\prime}$ as $k \rightarrow \infty$.

Let $\varphi \in \mathcal{C}_{0}^{\infty}$. We have

$$
\begin{aligned}
\int \varphi(x)\left\langle u_{p_{k}}(x), h\right\rangle_{H} d x & =\int\left\langle u_{p_{k}}(x), \varphi(x) h\right\rangle_{H} d x \\
& \rightarrow \int\langle u(x), \varphi(x) h\rangle_{H} d x,
\end{aligned}
$$

because $\int\langle\cdot(x), \varphi(x) h\rangle_{H} d x$ is a continuous linear functional on $\mathcal{H}^{t}\left(\mathbb{R}^{n}, H\right)$. Now, from the sequential continuity of $P(D)$ in $\mathcal{D}^{\prime}$, we conclude that the left-hand side of (19) converges to the left-hand side of (18) in $\mathcal{D}^{\prime}$. We shall prove the same for the right-hand sides, which means that

$$
\begin{aligned}
\int \varphi(x)\left\langle R_{p_{k}} F\left(x,\left(\partial^{\alpha} u_{p_{k}}(x)\right)_{|\alpha|<t-n / 2}\right), h\right\rangle_{H} d x \\
\rightarrow \int \varphi(x)\left\langle F\left(x,\left(\partial^{\alpha} u(x)\right)_{|\alpha|<t-n / 2}\right), h\right\rangle_{H} d x
\end{aligned}
$$

for any $\varphi \in \mathcal{C}_{0}^{\infty}$.

We show first that

$$
\begin{aligned}
& \left\langle R_{p_{k}} F\left(x,\left(\partial^{\alpha} u_{p_{k}}(x)\right)_{|\alpha|<t-n / 2}\right), h\right\rangle_{H} \\
& \rightarrow\left\langle F\left(x,\left(\partial^{\alpha} u(x)\right)_{|\alpha|<t-n / 2}\right), h\right\rangle_{H} \quad \text { for a.e. } x .
\end{aligned}
$$

Assume that $h \in H_{l}$ for some $l$. Then, for large $k$,

$$
\left\langle R_{p_{k}} F\left(x,\left(\partial^{\alpha} u_{p_{k}}(x)\right)_{|\alpha|<t-n / 2}\right), h\right\rangle_{H}=\left\langle F\left(x,\left(\partial^{\alpha} u_{p_{k}}(x)\right)_{|\alpha|<t-n / 2}\right), h\right\rangle_{H},
$$

hence (21) is true by the Carathéodory condition.

From (12) and (14), we have

$$
\left\|F\left(x,\left(\partial^{\alpha} u_{p_{k}}(x)\right)_{|\alpha|<t-n / 2}\right)\right\| \leq C(x)<\infty \quad \text { for a.e. } x .
$$

This implies (21) for all $h \in H$ (see [9], p. 121, Theorem 3). By the Lebesgue convergence theorem, formulas (12), (14) and (21) imply the convergence (20). The proof is complete.

EXAMPLE 1. We define a class of equations satisfying the assumptions of Theorem 1.

Let $P$ be a polynomial of $n$ variables and degree $T$ such that the polynomial $P(-i \partial)$ of the variable $\partial$ has real coefficients and satisfies (13). Let $F: \mathbb{R}^{n} \times \mathbb{R}^{m} \rightarrow H$ satisfy (14) and the Carathéodory condition in the sense of Theorem 1. Assume that there exist constants $0<a<2, L>0$ and 
nonnegative functions $f \in L^{2 / a}$ and $g \in L^{2 /(2-a)}$ such that

$$
\begin{aligned}
\left\langle v_{(0, \ldots, 0)}, F\left(x,\left(v_{\alpha}\right)_{|\alpha|<t-n / 2}\right)\right\rangle_{H} & \leq 0 \\
& \text { for }\left\|v_{(0, \ldots, 0)}\right\| \geq g(x) \text { and a.e. } x,
\end{aligned}
$$

and

$$
\begin{aligned}
& \left\|F\left(x,\left(v_{\alpha}\right)_{|\alpha|<t-n / 2}\right)\right\| \leq f(x)+L\left\|\left(v_{\alpha}\right)_{|\alpha|<t-n / 2}\right\|^{a} \\
& \quad \text { for }\left\|v_{(0, \ldots, 0)}\right\| \leq g(x) \text { and a.e. } x .
\end{aligned}
$$

Treating $H_{p}$ as $\mathbb{R}^{p}$, we obtain the necessary a priori bounds for solutions of equations (15) as in [4], Example 2.

Example 2. We now describe a more concrete example of the class described above.

Let $n=1, T=2, a=1, P(\xi)=\xi^{2}+b, b>0$, and $A: H \rightarrow H$ a linear, continuous, invertible operator. Suppose that $B: \mathbb{R} \times H \rightarrow H$ satisfies the Carathéodory condition in the sense of Theorem 1 and

$$
\|B(x, v)\| \leq h(x), \quad h \in L^{2}(\mathbb{R}) .
$$

Let $F(x, v)=-A^{*} A v+B(x, v)$. We have

$$
\begin{aligned}
\langle v, F(x, v)\rangle_{H} & =-\left\langle v, A^{*} A v\right\rangle+\langle v, B(x, v)\rangle \\
& =-\langle A v, A v\rangle+\langle v, B(x, v)\rangle \\
& \leq-C\|v\|^{2}+\|v\| h(x) \leq 0
\end{aligned}
$$

for $\|v\| \geq g(x):=h(x) / C$ with some constant $C>0$. Then condition (23) is satisfied. Condition (24) is satisfied for $L=0$ and

$$
f(x)=\left(\left\|A^{*} A\right\| / C+1\right) h(x) .
$$

Consider, for example, the following problem:

$$
-\frac{d^{2} u(x, t)}{d x^{2}}+u(x, t)=-u(x, t)+\psi\left(x, \int_{0}^{1} K(x, t, \tau) u(x, \tau) d \tau\right),
$$

where $K$ is measurable, $K(x, \cdot, \cdot) \in L^{2}([0,1] \times[0,1])$ for a.e. $x, \psi: \mathbb{R}^{2} \rightarrow \mathbb{R}$ is continuous and

$$
|\psi(x, y)| \leq h(x), \quad h \in L^{2}(x) .
$$

We look for $u \in \mathcal{H}^{1}\left(\mathbb{R}, L^{2}([0,1])\right)$ (we treat $u$ as the mapping $x \mapsto u(x, \cdot)$ ). We have

$$
P(\xi)=\xi^{2}+1
$$

and

$$
F(x, v)=-v+\psi\left(x, \int_{0}^{1} K(x, \cdot, \tau) v(\tau) d \tau\right) .
$$


The function $F$ satisfies the Carathéodory condition. In fact, the map

$$
L^{2}([0,1]) \ni v \mapsto \int_{0}^{1} K(x, \cdot, \tau) d \tau \in L^{2}([0,1])
$$

is linear and completely continuous (for almost all $x$ ), hence it transforms weakly convergent sequences to strongly convergent ones. The Nemytskiu operator

$$
L^{2}([0,1]) \ni v \mapsto \psi(x, v(\cdot)) \in L^{2}([0,1])
$$

is continuous by (25) (see for example [2], Proposition 1).

Remark 2. Note that a Hammerstein operator does not have so good properties as the operator $F$ defined in Example 2. Consider the operator

$$
G(v)=\int_{0}^{1} K(\cdot, \tau) \psi(\tau, v(\tau)) d \tau
$$

where

$$
K \in L^{2}([0,1] \times[0,1]), \quad|\psi(t, y)| \leq h(t)+|y|, \quad h \in L^{2}([0,1]) .
$$

Suppose that $G: L^{2}([0,1]) \rightarrow L^{2}([0,1])$ is sequentially continuous in the sense of the weak topology in $L^{2}([0,1])$. Then, for any $w \in L^{2}([0,1])$, the map

$$
G_{w}: v \mapsto\langle G(v), w\rangle_{L^{2}([0,1])}
$$

transforms weakly convergent sequences in $L^{2}([0,1])$ to convergent numerical ones.

Suppose that $\psi$ is differentiable with respect to the second variable and that $G_{w}: L^{2}([0,1]) \rightarrow \mathbb{R}$ satisfies the assumptions of the following theorem of Palmer (see [7]):

Let $X$ be a reflexive Banach space, $Y$ a Banach space and let $F: X \rightarrow Y$ be uniformly Fréchet differentiable on any ball in $X$. Then $F$ is sequentially continuous with the weak topology in $X$ and the strong topology in $Y$ if and only if the following two conditions are satisfied:

(i) for any $v \in X$ the Fréchet derivative $F^{\prime}(v)$ is a completely continuous linear operator,

(ii) the Fréchet derivative $F^{\prime}: X \rightarrow L(X, Y)$ (the space of linear continuous operators from $X$ into $Y$ ) is completely continuous.

We have

$$
G_{w}(v)=\int_{0}^{1} \int_{0}^{1} K(t, \tau) \psi(\tau, v(\tau)) w(t) d \tau d t=\int_{0}^{1} K_{w}(\tau) \psi(\tau, v(\tau)) d \tau
$$


where

$$
K_{w}(\tau):=\int_{0}^{1} K(t, \tau) w(t) d t .
$$

Compute the derivative

$$
G_{w}^{\prime}(v) \cdot h=\int_{0}^{1} K_{w}(\tau) \partial_{2} \psi(\tau, v(\tau)) h(\tau) d \tau .
$$

By the isomorphism $L\left(L^{2}([0,1]) ; \mathbb{R}\right) \cong L^{2}([0,1])$, we have

$$
G_{w}^{\prime}(v)=K_{w}(\cdot) \partial_{2} \psi(\cdot, v(\cdot)) .
$$

We conclude that the condition (ii) will not be satisfied if $G_{w}^{\prime}$ is not constant. In fact, any nonconstant superposition operator

$$
L^{2}([0,1]) \ni v \mapsto N(v):=\varphi(\cdot, v(\cdot)) \in L^{2}([0,1])
$$

does not transform bounded sets onto precompact ones. In fact, let $N\left(u_{1}\right) \neq$ $N\left(u_{2}\right)$ for some $u_{1}, u_{2} \in L^{2}([0,1])$. Let

$$
v_{k}(x):= \begin{cases}u_{1}(x) & \text { for } x \in\left[2^{-k} 2 p, 2^{-k}(2 p+1)[,\right. \\ u_{2}(x) & \text { for } x \in\left[2^{-k}(2 p+1), 2^{-k}(2 p+2)[,\right. \\ 0 & \text { for } x=1, p=0,1, \ldots, 2^{k-2} .\end{cases}
$$

The sequence $\left(v_{k}\right)$ is bounded in $L^{2}([0,1])$ but $N\left(v_{k}\right)$ has no subsequence which converges in $L^{2}([0,1])$.

4. Existence theorem for a system of equations. We formulate a theorem similar to Theorem 1 for systems of equations.

THEOREM 2. Let $H$ be a real infinite-dimensional separable Hilbert space, and $\left(e_{\gamma}\right)_{\gamma=1,2, \ldots}$ a complete orthonormal system in $H$. Let $H_{p}$ denote the space generated by the system $\left\{e_{\gamma}: \gamma=1, \ldots, p\right\}$ and let $R_{p}: H \rightarrow H_{p}$ be the orthonormal projector onto $H_{p}$. Let $P_{r}$ be polynomials of $n$ variables and degrees $T_{r}$ such that the polynomials $P_{r}(-i \partial)$ of the variable $\partial$ have real coefficients and satisfy

$$
1+|\xi|^{T_{r}} \leq C P_{r}(\xi), \quad \xi \in \mathbb{R}^{n}, r=1, \ldots, k,
$$

for some constant $C$. Let $t_{r} \in\left[0, T_{r}[, r=1, \ldots, k\right.$, and

$$
m:=\sum_{r=1}^{k} \sum_{0 \leq l<t_{r}-n / 2} n^{l} .
$$

Assume that $F: \mathbb{R}^{n} \times H^{m} \rightarrow H^{k}$ satisfies the Carathéodory condition of the following form: $F(x, \cdot)$ is sequentially continuous in the weak topologies of $H^{m}$ and $H^{k}$ for a.e. $x$ and $F\left(\cdot,\left(v_{\alpha}^{r}\right)_{|\alpha|<t_{r}-n / 2, r=1, \ldots, k}\right)$ is measurable for 
all $\left(v_{\alpha}^{r}\right)_{|\alpha|<t_{r}-n / 2, r=1, \ldots, k} \in H^{m}$. Assume that for any bounded set $K \subset$ $\mathbb{R}^{n} \times H^{m}$ there exists a function $h_{K} \in L^{2}\left(\mathbb{R}^{n}\right)$ such that

$$
\left\|F\left(x,\left(v_{\alpha}^{r}\right)_{|\alpha|<t_{r}-n / 2, r=1, \ldots, k}\right)\right\| \leq h_{K}(x)
$$

for $\left(x,\left(v_{\alpha}^{r}\right)_{|\alpha|<t_{r}-n / 2, r=1, \ldots, k}\right) \in K$ a.e. $x$. Assume that there is a sequence of open bounded sets $U_{1} \subset U_{2} \subset \ldots$ with $\bigcup U_{j}=\mathbb{R}^{n}$ and a constant $M$ such that no system

$$
\begin{aligned}
P_{l}(D) u^{l}=\lambda R_{p} F_{j}^{l}\left(x,\left(\partial^{\alpha} u^{r}\right)_{|\alpha|<t_{r}-n / 2, r=1, \ldots, k}\right), & \\
& \quad=1, \ldots, k\left(F=\left(F^{1}, \ldots, F^{k}\right)\right),
\end{aligned}
$$

has a solution in the set

$$
\left\{u=\left(u^{1}, \ldots, u^{k}\right) \in \underset{r=1}{\chi} \mathcal{H}^{t_{r}}\left(\mathbb{R}^{n}, H_{p}\right): \sum_{r=1}^{k}\left\|u^{r}\right\|_{\mathcal{H}^{t_{r}\left(\mathbb{R}^{n}, H\right)}}^{2}>M^{2}\right\}
$$

for $j=1,2, \ldots, \lambda \in[0,1], p=1,2, \ldots$ (The functions $F_{j}^{l}$ are defined as

$$
\begin{aligned}
F_{j}^{l}\left(x,\left(v_{\alpha}^{r}\right)_{|\alpha|<t_{r}-n / 2, r}=1, \ldots, k\right) & \\
& := \begin{cases}F^{l}\left(x,\left(v_{\alpha}^{r}\right)|\alpha|<t_{r}-n / 2, r=1, \ldots, k\right) & \text { for } x \in U_{j}, \\
0 & \text { for } \left.x \notin U_{j} .\right)\end{cases}
\end{aligned}
$$

Under these assumptions the system

$$
P_{l}(D) u^{l}=F^{l}\left(x,\left(\partial^{\alpha} u^{r}\right)_{|\alpha|<t_{r}-n / 2, r=1, \ldots, k}\right), \quad l=1, \ldots, k,
$$

has a solution $u \in \chi_{r=1}^{k} \mathcal{H}^{t_{r}}\left(\mathbb{R}^{n}, H\right)$ for which

$$
\sum_{r=1}^{k}\left\|u^{r}\right\|_{\mathcal{H}^{t_{r}\left(\mathbb{R}^{n}, H\right)}} \leq M
$$

Pr o of. Similar to the proof of Theorem 1.

ExAmPLE 3. One can construct an example analogous to Example 1 with the condition

$$
\begin{aligned}
& \left\langle v_{(0, \ldots, 0)}, F\left(x,\left(v_{\alpha}^{r}\right)|\alpha|<t_{r}-n / 2, r=1, \ldots, k\right)\right\rangle_{H^{k}} \leq 0 \\
& \qquad \text { for }\left\|v_{(0, \ldots, 0)}\right\| \geq g(x) \text { for a.e. } x
\end{aligned}
$$

instead of (23).

\section{References}

[1] S. N. Bernstein, Sur les équations du calcul des variations, Ann. Sci. École Norm. Sup. 29 (1912), 431-485.

[2] F. E. Browder, Nonlinear functional analysis and nonlinear integral equations of Hammerstein and Urysohn type, in: Contributions to Nonlinear Functional Analysis, Acad. Press, New York, 1971, 425-500. 
[3] P. Fijałkowski, On the equation $x^{\prime \prime}(t)=F(t, x(t))$ in the Sobolev space $H^{1}(\mathbb{R})$, Ann. Polon. Math. 53 (1991), 29-34.

[4] - On the solvability of nonlinear elliptic systems in Sobolev spaces, ibid. 56 (1992), 149-156.

[5] A. Granas, R. Guenther and J. Lee, Nonlinear boundary value problems for ordinary differential equations, Dissertationes Math. 244 (1985).

[6] L. Hörmander, The Analysis of Linear Partial Differential Operators, Springer, Berlin, 1983.

[7] K. J. Palmer, On the complete continuity of differentiable mappings, J. Austral. Math. Soc. 9 (1969), 3-4.

[8] B. Przeradzki, On the solvability of singular BVP's for second-order ordinary differential equations, Ann. Polon. Math. 50 (1990), 279-289.

[9] K. Yosida, Functional Analysis, Springer, Berlin, 1980.

INSTITUTE OF MATHEMATICS

UNIVERSITY OF ŁÓDŹ

S. BANACHA 22

90-238 ŁÓDŹ, POLAND

Reçu par la Rédaction le 28.10.1992

Révisé le 3.3 .1993 et 5.7.1993 